\title{
Performance Analysis of State-Free Geographic Forwarding Protocols for Wireless Sensor Networks
}

\author{
Idris Abubakar Umar $^{\# 1}$, Zurina M. Hanapi ${ }^{* 2}$ and Ali Idarous Adnan ${ }^{\# 3}$ \\ Department of Communication Technology ${ }^{123}$ \\ University Putra Malaysia., Selangor, Malaysia. \\ brossadiq@gmail.com ${ }^{1}$ \\ zurina@fsktm.upm.edu.my ${ }^{2}$ \\ aliadnn@gmail.com ${ }^{3}$
}

\begin{abstract}
Ideally in Wireless Sensor Network (WSN), the bandwidth (usually a single channel) and the radio transmission range are set to $250 \mathrm{Kbps}$ and $40 \mathrm{~m}$ respectively to ensure error free communication. However, dense deployment of sensor exposes them to various sources of problems such as transmission error and link failure, which eventually leads to high retransmission rate that results in increased congestion, overhead, and delay in the limited shared channel thus hindering network performance. This paper investigates via simulation, the effect of increasing traffic under various bandwidth capacity in a multi-hop network operated using a state free cross-layer based routing protocols, which perform the lazy binding technique when routing. Extensive experiments undertaken on the state-free protocols have shown that the low channel capacity yields higher packet delivery ratio when compared to the higher bandwidth capacity, which is more exposed to interference and congestion.
\end{abstract}

Keywords—routing, bandwidth, lazy binding, implicit geographic forwarding.

\section{INTRODUCTION}

The main task of a sensor network is to sense and cooperatively collect information of interest from a monitored region. The information is collected so that actions via an actuator or other means can be responded to in a timely manner. But due to constraints such as energy, bandwidth, memory, processing power, just to mention a few. Compromises have to be made to ensure the efficiency in response. During these communication processes, the routing protocols, which plays the communication coordinators, defines how messages are processed from one end of the network to another.

In this study, we selected two (2) state free geographic protocols. The protocols; Secured Implicit Geographic Forwarding(SIGF) and Dynamic Window Secured Implicit Geographic Forwarding (DWSIGF) exhibit lazy binding, a concept described by Blum et al. 2003 and He 2004, The binding technique enables the network to cope with elevated dynamics found in WSN, such that binding is done at the latest possible moment. Furthermore, the protocol ensures that communicating entities (nodes) are completely independent of the knowledge of the network's topology, hence, enabling a reduced cost in bandwidth utilization as the need for routing table upkeep has been eliminated. These features have made both SIGF and DWSIGF robust to topology shifts and less exposed to channel capacity problem [1], [3]-[5].

Furthermore, the protocols employ a cross-layer design feature that combines the network layer and the MAC layer to achieve robustness and fault tolerance so as to improve routing performance in the protocol[7]. The basic IEEE 802.11 DCF MAC which uses a direct sequence spread spectrum in $2.4 \mathrm{GHzthat}$ supports up to $2 \mathrm{Mbits} / \mathrm{s}$ and higher[8], is utilized in the MAC layer. It was originally created for a single access point scenario such that nodes that are within transmitting range of one another can avoid interference and improved efficiency.

Repeated use of the 802.11 DCF MAC in a multi-hop network is known to generate high overhead as a result of the generated control messages that are exchanged during its 4-way handshake (Request to Send - RTS, Clear to Send - CTS and Acknowledgement -ACK). The generated overhead is necessary for ensuring reliability in message delivery. However, the channel capacity fails to accommodate the needed data due to the substantial amount of overhead as the traffic becomes heavier. Data packets might be put into buffers and must wait to have access to the shared medium [7], [9]. This if allowed to prosper might result in increased delay as well as packet loss, which affects the entire performance of the network. This paper investigates via simulation the effect of increasing traffic flow under various bandwidth capacities using both state free protocols which perform lazy binding in path selection.

The rest of this paper is organized as follows. Section II reviews related works. Section III describes the protocols investigated. Section IV, present performance evaluation and results discussions. Section V concludes the paper. 


\section{RELATED WORKS}

In WSN, the most presented network topology considers densely deployed nodes, in which a few are dedicated to sensing the data of interest and propagating it at a constant bit rate(CBR), through multiple hops for delivery to a single or multiple sinks[10], [11]. SIGF and DWSIGF use this network topology for their operation. Thus, understanding of the traffic behavior and burden placed on intermediary nodes will add more knowledge on how to build an effective WSN where resources such as bandwidth capacity could be efficiently utilized.

The design in a low-bandwidth based radio model is such that it is able to accommodate the co-existence of communicating entities in the presence of interference. Interference by control messages employed by the MAC protocol for instance, at times of receiving CTS signals, nodes overhearing such signals would have to set their Network Allocation Vector (NAV) in accordance to the rules (802.11 timing semantics) on the basis of information received. This interferes with ongoing transmissions[8]. Mcouat, 2013 further explained that the separating distance between the interfering node and the receiving node helps drains the interfering nodes signal to a level that allows the receiving node to function normally within the low-bandwidth radio model as long as the interfering signal is less than the acceptable noise level. However, in such circumstances, it can be assumed that bandwidth capacity that exceeds the required placement and depending on the data rate propagated within the symmetric link might face difficulty in their normal workings.

He et al. 2007 explains a behavior observed in some geographic routing protocols (Implicit Geographic Forwarding and Greedy Perimeter Stateless Routing), stating that as traffic becomes heavy enough to congest a network, performance degrades due to limited intersecting routes suffering from the collision caused by nodes overhearing each other[12]. The limited intersecting routes points to the fact that for WSN to behave efficiently even in the presence of problems, one must consider the distance between the communicating entities also known as the degree of coverage[13], the message generated by the protocol (control messages) as well as the binding characteristics (lazy, eager or early binding). In an effort to fix the congestion problem some researchers adopted the use of multipath routing algorithms, these mitigated the congestion problem in the selected path at the expense of increased overall traffic overhead, delay and energy consumption [14].

The wide adoption of the contention-based 802.11DCF MAC protocol has helped in reducing the hidden terminal problem faced in shared infrastructure medium but it is worth noting that the continuous node to node transmission of feedback control signals (ACK)and broadcast signal can cause unlikeable changes behavior in traffic pattern and behavior even though reliability in message delivery is ensured, these hinders the channel capacity to cope, as these changes cumulate due to congestion and retransmission in the network[7], [9], [15].

There are still a number of ongoing researches centered on explaining the channel behavior on the different protocol designs. However, the boundary existing between the different layers and the plethora of information emanating from each layer is yet to produce clear and precise explanation between the modified layers in protocols. Thus, restrictive information in bit or pieces of the layer which a research is centered on is given. In this paper, we attempt to explain the channel behavior in SIGF and DWSIGF cross-layer state free protocols with lazy binding features.

\section{THE ROUTING PROTOCOLS}

The routing protocol is the functional activity coordinator in a network; it is responsible for moving information from one end of the network to another. As earlier mentioned this work is centered on SIGF and DWSIGF protocols that adopt the concept of lazy binding. Lazy binding as defined by He 2004 as" deferring mapping system physics (network topologies) into volatile states (route states), required by a certain operation as late as this operation allows" thus forwarding a packet as late as possible ensures delivery since the chances are packets are not delivered to nodes that fail or move out of range. This improves robustness and reliability in the protocols. Other properties of the protocols are further explained as stated below.

\section{A. Properties of SIGF and DWSIGF}

- Location based/opportunistic: These categories of protocols are known to have high throughputs, low latencies and are energy efficient. Also, their state free nature makes them independent of their network topology as once the position of the destination node is known to them, all operation revert to in-network processing.

- Multipath based routing: This kind of routing is employed in them to improve fault tolerance and reliability in the routing scheme. However, it is done at the expense of increased overhead and energy. Multipath based routing is also affected by interference when a communicating entity falls within the range of another entity, thereby reducing the available bandwidth capacity of each entity[6].

- Forwarding candidate selection scheme: SIGF and DWSIGF selection scheme is based on priority and randomness. The MAC used in the protocol is slightly modified, an Open-RTS (ORTS-broadcast in nature) is accompanied by a CTS signal. The ORTS sender sets up a Collection Window Time (CWT) to collect enough CTS responses from contending nodes. SIGF uses a fixed CWT while DWSIGF employs a random CWT to increase its dynamic properties as shown in table I. This timing response in the protocol causes an alteration, by increasing the delay in CTS response to the broadcast signal received. 
An appropriate relay node is chosen after the collected responses have being sampled and analyzed based on the selection scheme utilized:

o priority- otherwise known as greedy selection, as only the node that makes the maximum progress towards the destination is selected

- Randomness- where after collection of various CTS signal a node is selected randomly.

- Void avoidance mechanism: This mechanism is employed to avoid wasting the bandwidth due to failed transmission and retransmission. SIGF and DWSIGF are capable of shifting their angle of projection to a direction where the densities of the nodes are capable of ensuring an end-to-end delivery of the propagated message.

- Retransmission and congestion control mechanism: The standard 802.11 DCF mechanism is utilized in this aspect such that if a collision is detected. A node enters into a random backoff period which increases exponentially, after which the channel will have to be sensed for ongoing transmission. If the channel is found idle, retransmission ensues, otherwise, more delay is incurred. Buffers are used as congestion control mechanisms are used to mitigate packet loss in the network.

TABLE I. Protocol Classification

\begin{tabular}{|c|c|c|}
\hline Routing Protocol & Selection Scheme & Timing Dynamism \\
\hline SIGF-Priority & Priority & Constant \\
\hline SIGF-Random & Random & Constant \\
\hline DWSIGF-Priority & Priority & Random \\
\hline
\end{tabular}

Our goal is to analyze the performance of these protocols at different channel capacity while in increasing traffic flow at a constant bit rate. Details of the simulation parameter will be presented in the next section together with its discussion.

\section{PERFORMANCE EVALUATION}

To enable a fair comparison of the state-free routing protocols in Table I, we simulated all protocols using MATLAB. Each protocol's performance was tested under increasing traffic rate to determine the packet delivery ratio and message overhead at various bandwidth capacities. Table II present the simulation setting used for the experiment. The experiment considered two destination nodes receiving data from six source nodes, selected randomly from 196 nodes uniformly deployed in a 150x150 meter square terrain. The packet delivery which serves as a measure of the effectiveness of the node-to-node mapping for ensuring connectivity between the sources and destinations is shown in Fig.1-4, while Fig.5 - 8 shows the routing overhead generated when propagating the data from one end of the network to the other.

TABLE II. Simulation Parameters

\begin{tabular}{|l|l|}
\hline Terrain & 150 x 150 meters \\
\hline Number of Nodes & 196 \\
\hline Node placement & Grid $+\mathcal{N}(0,16)$ noise. \\
\hline Radio Range & 40 meters \\
\hline Radio Bandwidth & $100-300$ kbps (step 50) \\
\hline Payload Size & 32 bytes \\
\hline CTS Packet Size & 14 bytes \\
\hline ORTS Packet Size & 20 bytes \\
\hline ACK Packet Size & 14 bytes \\
\hline Traffic Load & 1 to 10 (Packet/Seconds) \\
\hline Simulation Length & 100 packet, 10 runs \\
\hline
\end{tabular}




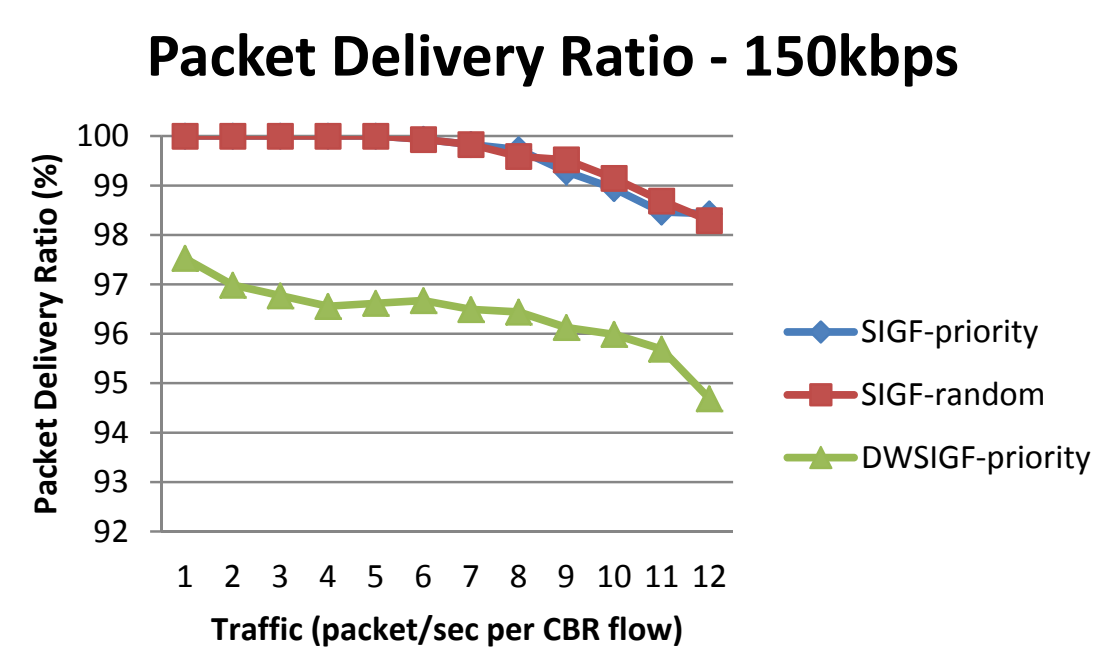

Fig. 1 Packet Delivery Ratio at 150Kbps

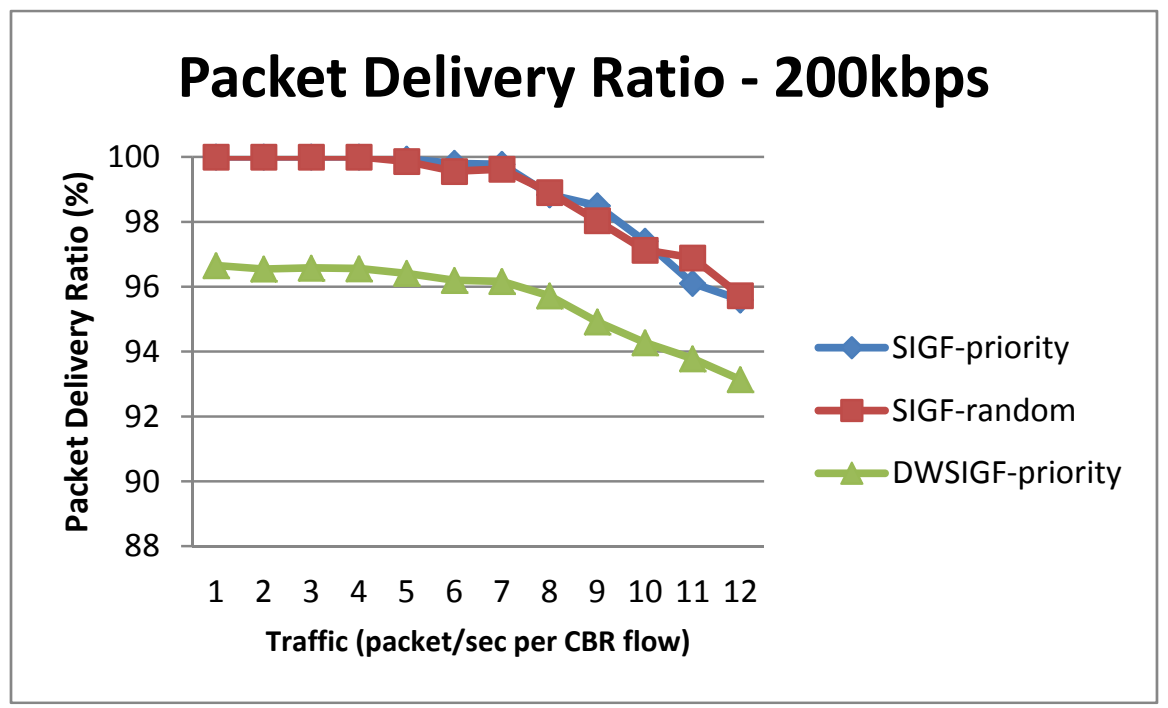

Fig. 2 Packet Delivery Ratio at 200Kbps

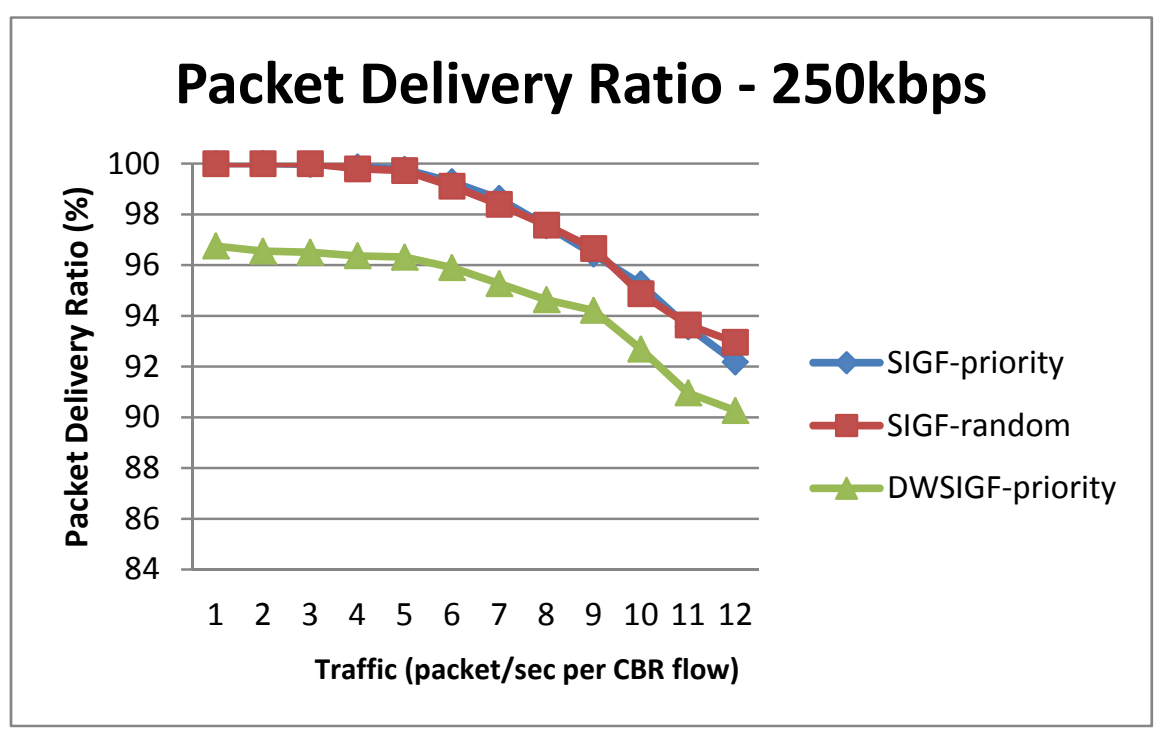

Fig. 3 Packet Delivery Ratio at 250Kbps 


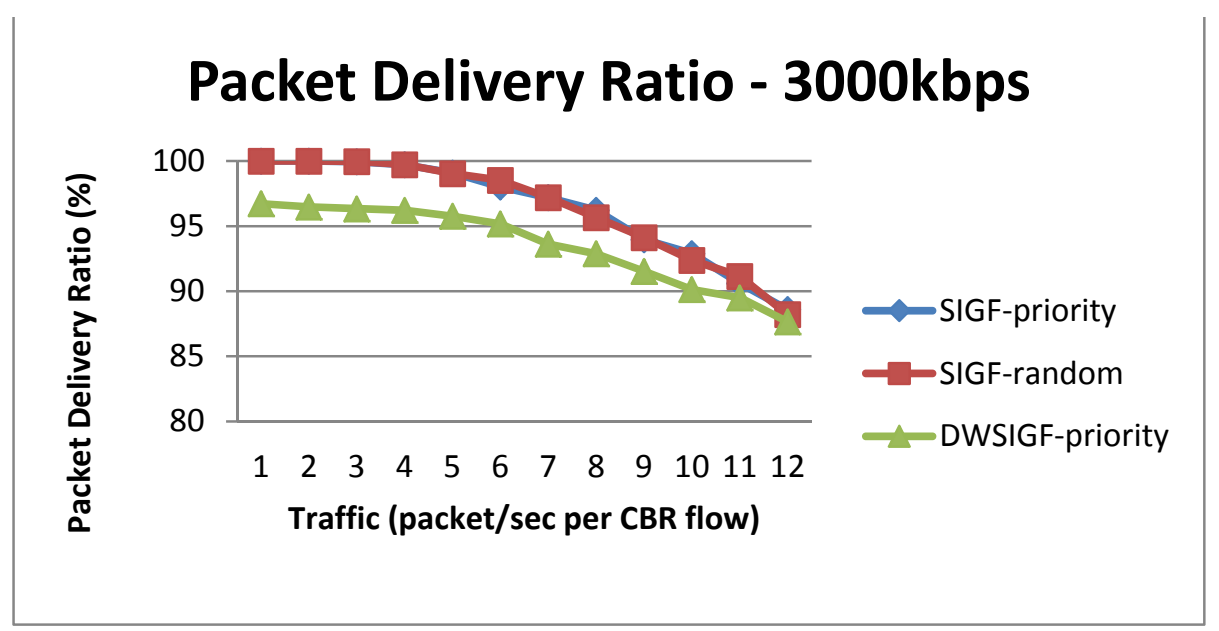

Fig. 4 Packet Delivery Ratio at 300Kbps

The random timing utilized in DWSIGF for the CTS response collection causes poor effectiveness in mapping (PDR) as shown in Fig.1- 4. This is because the random time assigned, when too small fails to collect any CTS response. These causes retransmission and makes it impossible for its PDR and message overhead to outperform both SIGF-P and SIGF-R as they utilize a fixed CWT as shown in all cases Fig1-4 and Fig. 5- 8.

The results obtained for PDR shown in Fig.1 to Fig.4 can be explained using the fireman, fire-hydrant, and hose-head. The hose-head control the pressure experienced when flushing the water towards the burning building. The smaller the hose-head outlet which in our case is the channel capacity, the higher the PDR which is the water flushed out the hose-head, the fire hydrant serves as the traffic controller (traffic pkt/sec). This is because, at that channel capacity (Fig 1), nodes are sufficient enough to provide connectivity and capacity to carry the desired traffic with minimum noise and interference level that allows the channel to function properly and at its best. As the traffic builds up ( $8 \mathrm{pkt} / \mathrm{sec})$, collision begins to rise thus making it a bit difficult to keep node connectivity which leads to a drop in the PDR as observed in fig 1 at $8 \mathrm{pkt} / \mathrm{sec}-12 \mathrm{pkt} / \mathrm{sec}$

Also, as the fireman increases the size of the hose-head outlet (increasing the channel capacity). The pressure at which the water is flushed also drops (PDR). This is because the frame size is bigger compared to the frame size passed through the smaller channel (150Kbps), and the bigger the size, the more prone it is to collision thus unable to maintain connectivity as the traffic is increased. This can be observed in Fig.2- 4, as PDR drop begins at $7 \mathrm{pkt} / \mathrm{sec}$ at $200 \mathrm{Kbps}, 6 \mathrm{pkt} / \mathrm{sec}$ at $250 \mathrm{Kbps}$ and $5 \mathrm{pkt} / \mathrm{sec}$ at $300 \mathrm{Kbps}$ respectively. It is also important to point out that the larger the capacity the higher interference signal received. And as long as the interference signal grows larger than the noise signal level, it makes it difficult to achieve the normal working output required from the channel.

Additionally, SIGF protocol has the lowest value for the message overhead incurred in sending data packets from one end of the network to the other (Fig. 5-8). This is because it maintains the smallest number of hops needed (in the case of SIGF-Priority) to traverse the network and its fixed CWT time provides enough time needed to collect CTS responses. This prevents subsequent retransmission which incurs increases message overhead. On the other hand, DWSIGF-Random increases as the bandwidth capacity is increased. The random CWT together with an increase in collision incurs retransmission, which further increases in the number of hops needed to reach the sink nodes. This subsequently increases the overhead as shown in Fig $5-8$. 


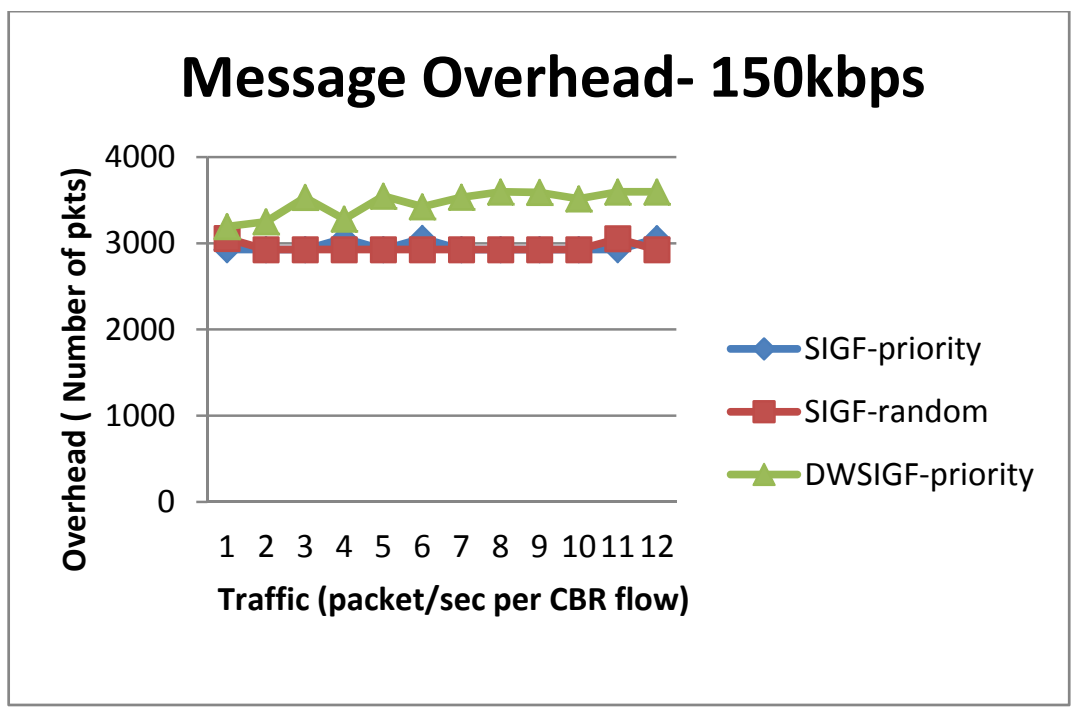

Fig. 5 Message Overhead at 150Kbps

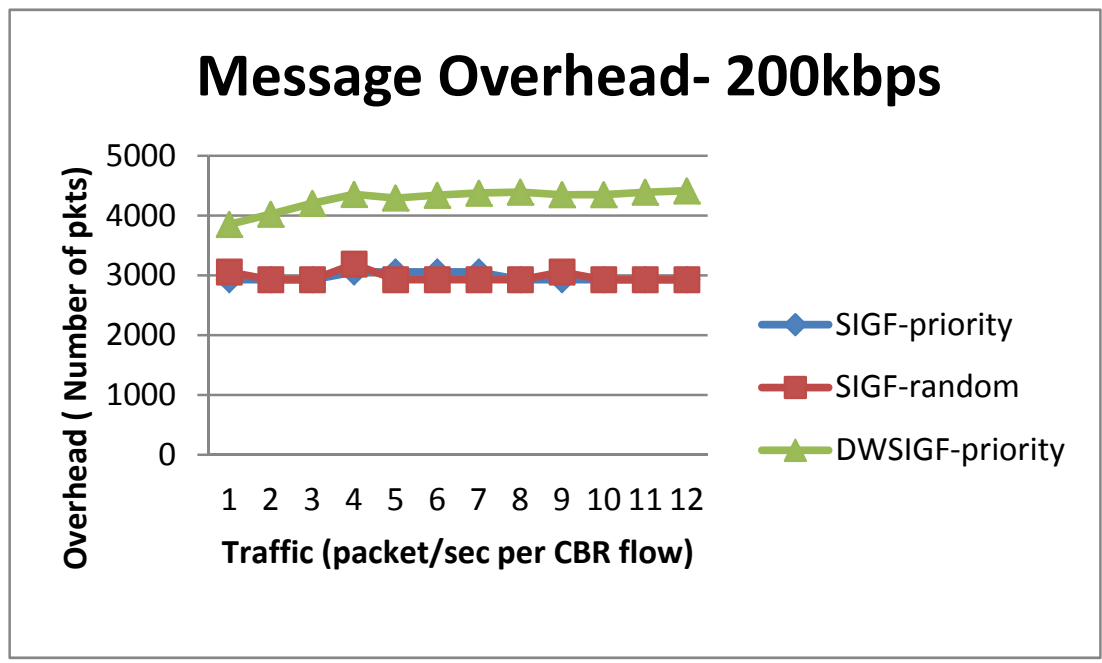

Fig. 6 Message Overhead at 200

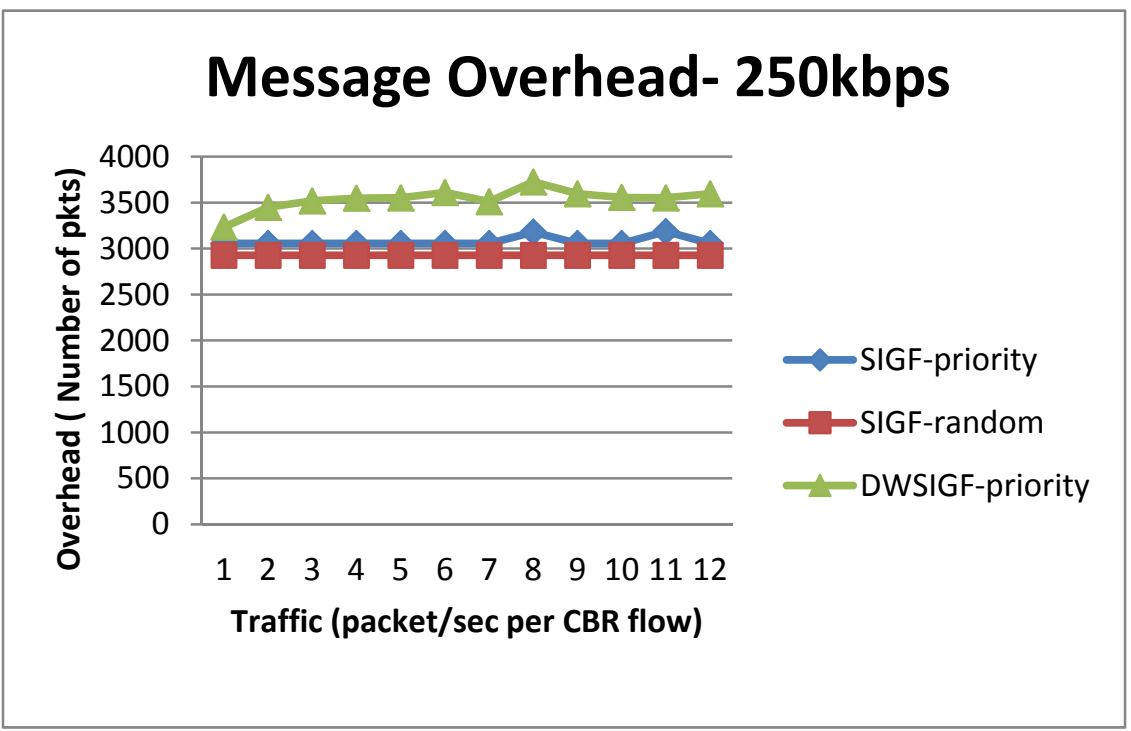

Fig. 7 Message Overhead at 250Kbps 


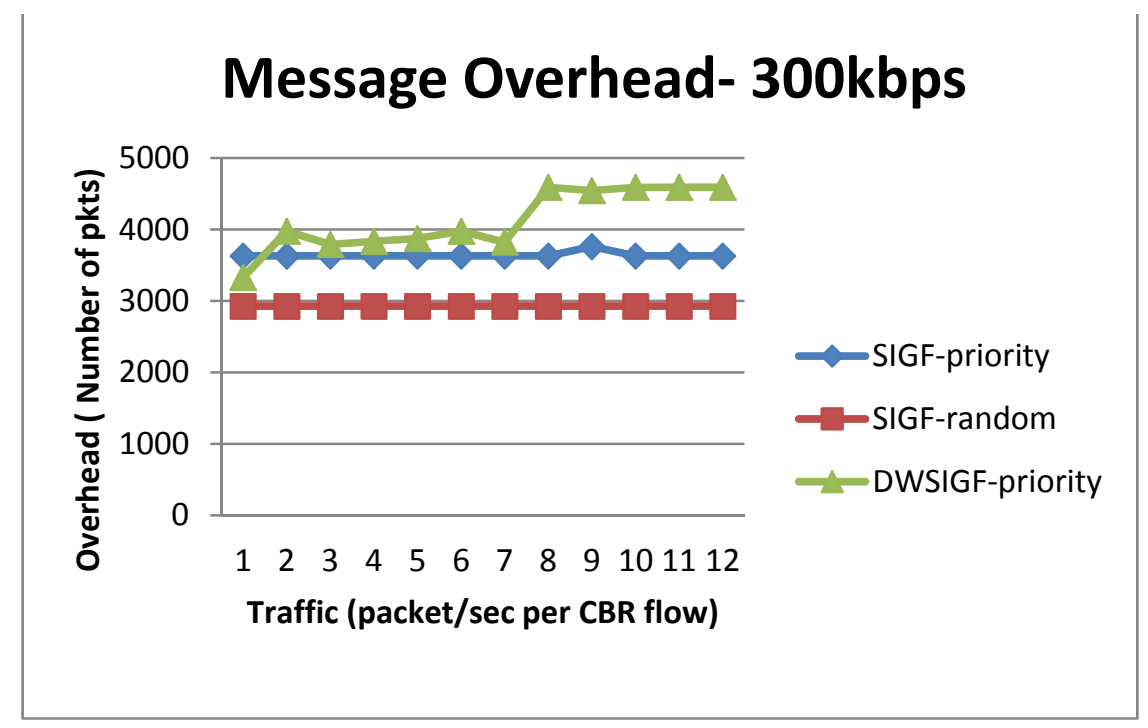

Fig. 8 Message Overhead at 300 kbps

\section{CONCLUSIONS}

In this paper, we analyzed the performance SIGF and DWSIGF routing protocols. The protocols utilize a lazy binding technique for forwarding. The analysis was based on protocols performance in terms of packet delivery ratio and message overhead. It can be inferred that a smaller channel capacity can best be utilized to propagate messages from one end of the network to another. This is due to the fact that frames traversing the channel are reduced to a much smaller size which enables easier mapping and reduces the congestion level. However, with a larger channel, the frame sizes are barely reduced and the larger frames are more prone to congestion (leading to packet drop) which in turn calls for retransmission of lost packets, which subsequently increases the message overhead.

\section{REFERENCES}

[1] B. Blum, T. He, S. Son, and Jack Stankovic, "IGF: A State-Free Robust Communication Protocol for Wireless Sensor Networks," Tech. Report, Dep. Comput. Sci. Univ. Virginia, 2003.

[2] T. He, "Hermes: A Composable Wireless Sensor Network Architecture for Dynamic , Energy and Spatiotemporal Awareness," 2004.

[3] A. D. Wood, L. Fang, and J. A. Stankovic, "SIGF : A Family of Configurable , Secure Routing Protocols for Wireless Sensor Networks," in Proceedings of the fourth ACM workshop on Security of ad hoc and sensor networks, 2006, pp. 35-48.

[4] Z. M. Hanapi, M. Ismail, K. Jumari, M. Mahdavi, and A. R. Protocols, "Forwarding Routing for Wireless Sensor Network," pp. 173$179,2009$.

[5] V. A. Dahat, V. A. Bhoyar, and V. A. Kalaskar, “A Review on State Free Routing Protocol For Wireless Sensor Network," Int. J. Pure Appl. Res. Eng. Technol., vol. 1, no. 8, pp. 349-360, 2013.

[6] K. Liu, A. Majeed, and N. B. Abu-ghazaleh, "TARMAC: Traffic-Analysis Resilient MAC Protocol for Multi-Hop Wireless Networks," 2006.

[7] G. Wu, F. Xia, L. Yao, Y. Zhang, and Y. Zhu, "A hop-by-hop cross-layer congestion control scheme for wireless sensor networks," J. Softw., vol. 6, pp. 2434-2440, 2011.

[8] M. Cesana, D. Maniezzo, P. Bergamo, and M. Gerla, "Interference aware (IA) MAC: an enhancement to IEEE802.11b DCF," 2003 IEEE 58th Veh. Technol. Conf. VTC 2003-Fall (IEEE Cat. No.03CH37484), vol. 5, pp. 2799-2803, 2003.

[9] W. W. Han, H. Park, and Y. M. Kim, "Dynamic Scheduling Protocol for Aggregation for Telematics Intersection Safety System (TISS)," 2007, pp. 163-172.

[10] Q. Wang, "Traffic Analysis \& Modeling in Wireless Sensor Networks and Their Applications on Network Optimization and Anomaly Detection.," Netw. Protoc. Algorithms, vol. 2, no. 1, pp. 74-92, 2010.

[11] M. S. Elsersy, "Balancing Energy Consumption and Average End-to-End Delay for Optimum Routing Protocol in Wireless Sensor Network," M.Sc thesis, Department of Electrical and Computer Engineering, Calgary Alberta, 2013.

[12] T. He, B. M. Blum, Q. Cao, J. a. Stankovic, S. H. Son, and T. F. Abdelzaher, "Robust and timely communication over highly dynamic sensor networks," Real-Time Syst., vol. 37, no. 3, pp. 261-289, Aug. 2007.

[13] I. Demirkol, C. Ersoy, and F. Alagöz, "MAC Protocols for Wireless Sensor Networks : a Survey," IEEE Commun. Mag., vol. 44, no. 4, pp. 115-121, 2006.

[14] S. S. K. Singh and M. M. Singh, "Energy Efficient Transmission Error Recovery for Wireless Sensor Networks," Int. J., vol. 3, no. 4, pp. 89-104, 2010.

[15] B. Hull, K. Jamieson, and H. Balakrishnan, "Bandwidth Management in Wireless Sensor Networks," in Proceedings of the 1st international conference on Embedded networked sensor systems. ACM, 2003.

[16] Joanna Mcouat, Wireless Sensor Networks: Principles, Design and Applications Springer Science \& Business Media Publishing, 2013 


\section{AUTHOR PROFILE}

Idris Abubakar Umar Received his B.Eng. in Computer Engineering from Bayero University Kano, Nigeria in 2008, M.Sc. In Computer Networks from University Putra, Malaysia in 2013. He is currently a Ph.D. student in Computer Networks in University Putra, Malaysia. His research interests focus on protocol development, security, cloud computing and Internet of things.

Zurina Mohd Hanapi Received her B.Sc.in Computer and Electronic Systems from the University of Strathclyde, Glasgow, UK in 1999, M.Sc. Computer and Communication System Engineering from University Putra Malaysia (UPM), in 2004 and Ph.D. from the University Kebangsaan Malaysia (UKM) in 2011. She is currently an Associate Professor in the Department of Wireless and Communication Technology, Faculty of Computer Science and Information Technology, UPM. Her research interests are in protocol development, security, wireless technologies, and cyber-physical systems.

Ali Idarous Adnan Received his B.Eng. in Computer Engineering from Vladimir State University, Russia in 2004, M.Sc. In Computer Engineering from Vladimir State University, Russia in 2006. He is currently a Ph.D. student in Computer Networks in University Putra, Malaysia. His research interests focus on wireless sensor networks, wireless communication protocols, network and security, and Internet of things. 